\title{
SIMPLE GROUPS ARE SCARCE
}

\section{LARRY DORNHOFF}

Even gross limits on the frequency of occurrence of finite simple groups seem to be lacking in the mathematical literature. The following theorem is offered as a first step in this direction.

I am indebted to E. L. Spitznagel for discussions leading to the formulation of this problem.

THEOREM 1. Let $r(x)$ denote the number of integers $n \leqq x$ such that every group of order $n$ has a normal Sylow subgroup. Then $\lim _{x \rightarrow \infty}(r(x) / x)=1$.

The proof requires only Sylow's theorem from group theory, plus the following two results from number theory. $[x]$ denotes the greatest integer in the number $x$.

THEOREM 2 [1, p. 356]. Let $f(n)$ denote the number of prime factors of $n$ (distinct or not). For any $\delta>0$, there exists $x_{0}$ such that, for all $x \geqq x_{0}$, the number of integers $n \leqq x$ satisfying $|f(n)-\log \log n|>(\log$ $\log n)^{1 / 2+\delta}$ is less than $\delta x$.

THEOREM 3 [1, p. 351]. There is a constant $B$ such that for any $\delta>0$, there exists $x_{0}$ such that $x \geqq x_{0}$ implies

$$
\left|\sum_{p \leq x} \frac{1}{p}-\log \log x-B\right|<\delta .
$$

(Here the letter $p$ is restricted to run through primes only.)

Proof of Theorem 1. Let $\epsilon>0$ be assigned. We wish to find $X_{0}$ such that for all $X \geqq X_{0}, r(X) \geqq(1-\epsilon) X$. Choose $X_{0}$ so large that the following conditions are all satisfied:

(1) If $x \geqq\left(X_{0}\right)^{1 / 2}-1$, then the number of integers $n \leqq x$ with more than $\log \log n+(\log \log n)^{8 / 4}$ prime factors is $<\epsilon x / 4$.

(2) If $x \geqq X_{0}$, then

$$
\frac{\log \log x-\log \log \log x-4 \log 2}{\log \log x+(\log \log x)^{3 / 4}+1}>1-\frac{\epsilon}{4} .
$$

(3) If $x \geqq X_{0}$, then $\log x>(1-\epsilon / 4)^{-1} \cdot(4 / \epsilon)$.

(4) If $x \geqq \log X_{0}$, then

Received by the editors March 9, 1967. 


$$
\left|\sum_{p \leq x} \frac{1}{p}-\log \log x-B\right|<\log 2 .
$$

(5) If $x \geqq\left(X_{0}\right)^{1 / 2}$, then $(1-\epsilon / 4) x<[x]$.

Now choose $X \geqq X_{0}$ and let $\Im$ denote the set of integers $n \leqq X$ such that every group of order $n$ has a normal Sylow subgroup. We shall show that the order of $J$ is at least $(1-\epsilon) X$, as required.

Let $\rho$ be the set of all primes $p$ satisfying $(\log X)^{2}<p \leqq X^{1 / 2}$. If $p$ is "in $\odot$, let $M_{p}$ denote the set of multiples of $p$ less than or equal to $X$; thus $M_{p}=\{p, 2 p, \cdots,[X / p] p\}$. Then $1+p$ divides at most $X / p^{2}$ members of $M_{p}, 1+2 p$ divides at most $X / 2 p^{2}$ members of $M_{p}$, etc. We find that at most

$$
\frac{X}{p^{2}} \sum_{n \leqq X / p^{2}} \frac{1}{n}<\frac{X}{p^{2}}\left(\log \frac{X}{p^{2}}+1\right)
$$

members of $M_{p}$ are divisible by a number of form $1+k p, k>0$.

Let $M_{p}^{\prime}$ consist of those numbers $n$ in $M_{p}$ such that $n$ has at most $\log \log n+(\log \log n)^{3 / 4}+1$ prime divisors. By (1), the order of $M_{p}^{\prime}$ is at least $(1-\epsilon / 4)[X / p]$. Hence, using Sylow's theorem, the order of $M_{p}^{\prime} \cap J$ is at least $(1-\epsilon / 4)[X / p]-\left(X / p^{2}\right)\left(\log \left(X / p^{2}\right)+1\right)$.

Any integer $n \leqq X$ is in $M_{p}^{\prime}$ for at most $\log \log X+(\log \log X)^{3 / 4}+1$ values of $p$. Using this fact, (5), (3), and (4) successively, we see that the order of $J$ is at least

$$
\begin{aligned}
& \frac{\sum_{p \in \mathcal{P}}\left\{\left(1-\frac{\epsilon}{4}\right)\left[\frac{X}{p}\right]-\frac{X}{p^{2}}\left(\log \frac{X}{p^{2}}+1\right)\right\}}{\log \log X+(\log \log X)^{3 / 4}+1} \\
& \geqq\left(1-\frac{2 \epsilon}{4}\right) \frac{\sum_{p \in \odot} \frac{X}{p}\left(1-\frac{1}{1-\epsilon / 4} \frac{\log X}{p}\right)}{\log \log X+(\log \log X)^{3 / 4}+1} \\
& \geqq\left(1-\frac{3 \epsilon}{4}\right) X \frac{\sum_{p \in \mathcal{P}} \frac{1}{p}}{\log \log X+(\log \log X)^{3 / 4}+1} \\
& \geqq\left(1-\frac{3 \epsilon}{4}\right) X \frac{\log \log X^{1 / 2}-\log \log (\log X)^{2}-2 \log 2}{\log \log X+(\log \log X)^{3 / 4}+1} .
\end{aligned}
$$

Now $\log \log X^{1 / 2}=\log \log X-\log 2$ and $\log \log (\log X)^{2}=\log \log \log X$ $+\log 2$, so the order of $J$ is at least $(1-\epsilon) X$ by (2). Q.E.D. 
This proof is based on the "relatively large" primes between $(\log X)^{2}$ and $X^{1 / 2}$. A proof cannot be based on the "very large" primes greater than $X^{1 / 2}$. The reason why is contained in Theorem 4 below, which may be of independent number-theoretic interest.

In the following, $\pi(x)$ denotes the number of primes less than or equal to $x$. We use the fact that $\sum_{n \leqq x}(1 / n)-\log x$ approaches a constant limit (Euler's constant) as $x \rightarrow \infty$ and also the prime number theorem $\lim _{x \rightarrow \infty}(\pi(x) /(x / \log x))=1$.

THEOREM 4. If $p(x)$ is the number of integers $n \leqq x$ with a prime divisor greater than $n^{1 / 2}$, then $\lim _{x \rightarrow \infty}(p(x) / x)=\log 2$.

Proof. Let $\epsilon>0$ be assigned. We shall show that there exists $N_{0}$ such that for all integers $N \geqq N_{0}$,

$$
|(p(2 N)-p(N))-N \log 2|<\epsilon N .
$$

This is sufficient. We may assume $\epsilon<\frac{1}{2}$.

We note that $\lim _{x \rightarrow 1^{+}}+((1 /(x-1)) /(1 / \log x))=1$, but $x /(x-1)$ $>1 / \log x$ for $x>1$. Hence we may choose $k>1$ with $((k-1) / k)$ $\cdot(1 / \log k)>1-\epsilon / 6$ and $k<1+\epsilon / 48$. We also choose $\tau$ such that $\tau /(k-1)=\epsilon / 6$.

Note that by the prime number theorem, as $x \rightarrow \infty, \pi(k x)-\pi(x)$ is asymptotic to

$$
\frac{k x}{\log k x}-\frac{x}{\log x}=\frac{x}{\log x}\left(k \cdot \frac{\log x}{\log k+\log x}-1\right),
$$

and so asymptotic to $(k-1) x / \log x$.

Choose $N_{0}$ sufficiently large so that

(6) For $M \geqq\left(N_{0}\right)^{1 / 2} / k, \quad(k-1-\tau) M / \log M \leqq \pi(k M)-\pi(M) \leqq$ $(k-1+\tau) M / \log M$.

(7) For $N \geqq N_{0}, \pi(2 N)-\pi(N)<(\epsilon \cdot \log 2 / 6) N$.

(8) If $N \geqq N_{0}$ and $s=\left[\log _{k} N^{1 / 2}\right]$, then $1-\epsilon / 6<(s-1) / s$.

(9) With $s$ as in (8), we have $\mid 1 / s+1 /(s+1)+\cdots+1 /(2 s-1)$ $-\log 2 \mid<\epsilon \cdot \log 2 / 6$.

Let $N$ be any integer greater than or equal to $N_{0}$, and define integers $L$ and $T$ by $k^{L} \leqq N^{1 / 2}, k^{L+1}>N^{1 / 2}, k^{T} \leqq(2 N)^{1 / 2}, k^{T+1}>(2 N)^{1 / 2}$. If $M$ is any number $M \geqq N^{1 / 2}$, consider primes $p$ satisfying $M<p$ $\leqq k M$. There are $\pi(k M)-\pi(M)$ such primes. If $p$ is such, then the number of multiples of $p$ between $N$ and $2 N$ is at least $[N / k M]$ and not more than $N / M+1$.

By choosing $M=k^{t} \cdot(2 N)^{1 / 2}, t=0,1, \cdots, T-1$, and using (6), we see that 
(10) $p(2 N)-p(N) \geqq \sum_{t=0}^{T-1}(k-1-\tau) \frac{k^{t} \cdot(2 N)^{1 / 2}}{\log k^{t} \cdot(2 N)^{1 / 2}}\left(\frac{N}{k^{t+1}(2 N)^{1 / 2}}-1\right)$.

By choosing $M=k^{t} \cdot N^{1 / 2}, t=0,1, \cdots, L$, we also see that

$$
\begin{aligned}
p(2 N)-p(N) \leqq & \sum_{t=0}^{L}(k-1+\tau) \frac{k^{t} \cdot(N)^{1 / 2}}{\log k^{t} \cdot(N)^{1 / 2}}\left(\frac{N}{k^{t} \cdot(N)^{1 / 2}}+1\right) \\
& +\pi(2 N)-\pi(N) .
\end{aligned}
$$

Using (6) and cancellation, (10) becomes

$$
\begin{aligned}
p(2 N)-p(N) \geqq & \frac{k-1-\tau}{k-1} \cdot \frac{k-1}{k} N \cdot \sum_{t=0}^{T-1} \frac{1}{\log (2 N)^{1 / 2}+t \log k} \\
& -(\pi(2 N)-\pi(N)) .
\end{aligned}
$$

Using (7) and the definitions of $\tau$ and $k$, we get

$$
\begin{aligned}
p(2 N)-p(N) \geqq & \left(1-\frac{2 \epsilon}{6}\right) \log k \cdot N \cdot \sum_{t=0}^{T-1} \frac{1}{\log (2 N)^{1 / 2}+t \log k} \\
& -\frac{\epsilon \cdot \log 2}{6} N .
\end{aligned}
$$

Now $k^{T} \leqq(2 N)^{1 / 2}$. Pick $k_{0}$ such that $k_{0}^{T}=(2 N)^{1 / 2}$. Since $k_{0} \geqq k$, we may replace $\log k$ by $\log k_{0}$ in the summation of (12) and preserve the inequality. We get

$$
\begin{aligned}
p(2 N)-p(N) & \geqq\left(1-\frac{2 \epsilon}{6}\right) \log k \cdot N \cdot \sum_{t=0}^{T-1} \frac{1}{(T+t) \log k_{0}}-\frac{\epsilon \cdot \log 2}{6} N \\
& =\left(1-\frac{2 \epsilon}{6}\right) \frac{\log k}{\log k_{0}} \cdot N \cdot \sum_{t=0}^{T-1} \frac{1}{T+t}-\frac{\epsilon \cdot \log 2}{6} N .
\end{aligned}
$$

Since $k^{T+1}>(2 N)^{1 / 2}$, we use (8) to see that

$$
\frac{\log k}{\log k_{0}}>\frac{(1 /(T+1)) \log (2 N)^{1 / 2}}{(1 / T) \log (2 N)^{1 / 2}}=\frac{T}{T+1}>1-\frac{\epsilon}{6} .
$$

By (9),

$$
\sum_{t=0}^{T-1} \frac{1}{T+t}>\log 2-\frac{\epsilon \cdot \log 2}{6}=\log 2\left(1-\frac{\epsilon}{6}\right) .
$$

Therefore we conclude that

$$
\begin{aligned}
p(2 N)-p(N) & >(1-4 \epsilon / 6) \log 2 \cdot N-(\epsilon \cdot \log 2 / 6) N \\
& >(1-\epsilon) \log 2 \cdot N .
\end{aligned}
$$


Now $k-1+\tau<2(k-1-\tau)$. Hence, using (11), (6), and the facts $k<1+\epsilon / 48, \log 2>\frac{1}{2}$, we easily get

$$
\begin{aligned}
p(2 N)-p(N) \leqq & \sum_{t=0}^{L-1}(k-1+\tau) \frac{k^{t} \cdot N^{1 / 2}}{\log k^{t} \cdot N^{1 / 2}} \cdot \frac{N}{k^{t} \cdot N^{1 / 2}} \\
& +3(\pi(2 N)-\pi(N))+\frac{\epsilon \cdot \log 2}{6} N \\
\leqq & \frac{k-1+\tau}{k-1} \cdot \frac{k-1}{k} \cdot k N \cdot \sum_{t=0}^{L-1} \frac{1}{\log N^{1 / 2}+t \log k} \\
& +\frac{4 \epsilon \cdot \log 2}{6} N .
\end{aligned}
$$

$k^{L} \leqq N^{1 / 2}$. Hence $L \log k \leqq \log N^{1 / 2}$, and the inequality is preserved if we replace $\log N^{1 / 2}$ by $L \log k$. Therefore

$$
\begin{aligned}
p(2 N)-p(N) & \leqq\left(1+\frac{\epsilon}{6}\right)\left(1+\frac{\epsilon}{48}\right) \frac{\log k}{\log k} N \cdot \sum_{t=0}^{L-1} \frac{1}{L+t}+\frac{4 \epsilon \cdot \log 2}{6} N \\
& \leqq\left(1+\frac{\epsilon}{48}\right)\left(1+\frac{\epsilon}{6}\right) N \log 2+\frac{4 \epsilon \cdot \log 2}{6} N \\
& <(1+\epsilon) N \log 2 .
\end{aligned}
$$

This together with (13) gives the result.

AdDEd IN Proof. P. T. Bateman has kindly pointed out that Theorem 4 is a special case of several results mentioned in Math. Rev. 34 (1967), \#5770.

\section{REFERENCE}

1. G. H. Hardy and E. M. Wright, An introduction to the theory of numbers, 4 th ed., Clarendon Press, Oxford, 1960.

YALE UNIVERSITY 